\title{
Bio-Functional Nanodiamond Restorative Materials Containing Bio-Additives: In Vitro Approach
}

\author{
Victoria Tamara Perchyonok ${ }^{1 *}$, Tatiana Souza ${ }^{2}$, Rafael Felliti ${ }^{3}$, Shengmiao Zhang4, \\ Sias Grobler 5 \\ ${ }^{1}$ VTPCHEM PTY LTD, Southport, Australia \\ ${ }^{2} \mathrm{~S} 3$, Logan, Australia \\ ${ }^{3}$ Colonia 1181 apt 302, Montevideo, Uruguay \\ ${ }^{4}$ School of Material Science and Engineering, East China University of Science and Technology, Shanghai, China \\ ${ }^{5}$ Oral and Dental Research Institute, Faculty of Dentistry, University of the Western Cape, Cape Town, \\ South Africa \\ Email: ${ }^{*}$ tamaraperchyonok@gmail.com
}

Received 30 March 2015; accepted 9 May 2015; published 12 May 2015

Copyright (C) 2015 by authors and Scientific Research Publishing Inc.

This work is licensed under the Creative Commons Attribution International License (CC BY). http://creativecommons.org/licenses/by/4.0/

c) (i) Open Access

\section{Abstract}

Restorative materials in the new era aim to be "bio-active", bio-functional and long-lasting. As a part of our continuous interest of developing functional dual action restorative materials capable of being "bio-active" functional restorative materials, we designed and evaluated several novel nano-diamond:chitosan containing hydrogels as a prototype of molecular scaffold materials capable of free radical defense action containing propolis extract (antioxidant containing material) from 3 different regions namely, Green Brazilian Propolis, Uruguan Propolis and Australian Propolis. We evaluated the physical properties, bonding to dentin as well as test the bioadhesion of the newly designed materials in order to access the suitability of these prototype materials as suitable restorative materials, evaluated total phenol capacity of the materials as well as free radical defense capacity using BSA protein model as a quick and effective in vitro model. The hydrogels were prepared as previously reported by our protocol. The physico-chemical features including surface morphology (SEM), release behaviors, stability of the antioxidant-chitosan-nanodiamond and the effect of the hydrogels on the shear bond strength of dentin were measured. Structural investigations of the reactive surface of the hydrogel are reported. Bio-adhesive studies were performed in order to assess the suitability of these designer materials. Free radical defence and performance of the materials was evaluated using BSA and Folin Ciocalteu assay. Results: Within the limitations of the study design chitosan-antioxidant hydrogels are suitable materials for functional restorative and periodontal applications in vitro. The addition of propolis extracts nano-diamond chito-

\footnotetext{
${ }^{*}$ Corresponding author.
} 
san prototype delivery system had a beneficial effect on the design of the hydrogel by increasing bioadhesive properties of the newly prepared hydrogels, increasing free radical defense properties of the biomaterials as well as increasing the dentin bond strength of the newly designed hydrogels. Conclusion: The added benefits of the chitosan treated hydrogels involved positive influence on the naproxen release as well as increased dentin bond strength as well as demonstrating good antimicrobial properties and enhanced antioxidant stability. The therapeutic polymer approach described here has a potential to provide clinical benefit, through the use of "designer" adhesive restorative materials with the desired properties.

\section{Keywords}

Therapeutic Polymers, Adhesives, Chitosan, Hydrogels, Propolis, Melatonin, Naproxen, Antimicrobial, Dentin Bonding, Antioxidants, Bioactive

\section{Introduction}

Restorative materials in the new era aim to be "bio-active", bio-functional and long-lasting. Nanodiamonds (NDs) are carbon nanoparticles which in recent years have cemented its role in the unique functional naomaterials due to their superior physical properties as well as the advantages of nanomaterials, such as small size, large surface area, and high adsorption. The new area of research has been created due to numerous reports of application of nanodiamond-based film to build drug delivery systems in medicine [1]-[3].

Propolis was used at the time of Egyptian and Greek civilizations which recognized its healing qualities. Hippocrates, the founder of modern medicine, used it for healing sores and ulcers internally and externally. The word propolis (Russian Penicillin) is derived from the Greek word "pro" before, polis "city" or defender of the city. This non-toxic resinous substance was classified into 12 types according to physicochemical properties and related to geographic locations; however, the botanical origin of only three types was identified [4].

Propolis has been used for treating different diseases and inflammatory conditions as both local and systemic applications. In dentistry propolis have shown efficacy and application in every area of conventional dentistry and there for gave us an important scope for development and evaluation of novel chitosan:nanodiamond bio-functional restorative materials [5].

As a part of our continuous interest of developing functional dual action restorative materials capable of being "bio-active" and long-lasting, we design and evaluate novel chitosan:nanodiamond hydrogels as functional additive prototypes for further development of "dual function restorative materials," with the build in capability of secondly to determine their effect on the dentin bond strength of a composite and thirdly evaluate the bio-adhesive capability of newly designed hydrogels as a "molecular prototype of the site of free radical attack in vitro.

\section{Materials and Methods}

Chitosan (Aldrich, Australia), glycerol (Sigma, USA), glacial acetic acid (E. Merck, Germany) were used as received. The degree of de-acetylation of typical commercial chitosan used in this study is $87 \%$. Chitosan with molecular weight $2.5 \times 10^{3} \mathrm{KD}$ was used in the study. The isoelectric point is $4.0-5.0$. Propolis (Aurora Pharmaceuticals, Australia), Green Propolis (Brazilian Propolis) and Uruguyan Propolis were used as received. Nanodiamonds where purchased from Ebersoles, (25 carats, 5 grams, size (0 - 2 microns), Grit 14,000) and used as received

\subsection{Preparation of Various Chitosan:Nanodiamond Hydrogels Gels: General Protocol}

The therapeutic agent such as propolis containing gel was prepared by dispersion of $0.2 \mathrm{gm}$ in glycerol $(5 \% \mathrm{w} / \mathrm{w})$ $(1 \mathrm{ml})$ using a mortar and a pestle following the earlier reported generic protocol. [6] Ten milliliters of glacial acetic acid $(3 \% \mathrm{w} / \mathrm{w})$ was then added with continuous mixing and finally nanodiamond:chitosan (10\% nanodiamond:chitosan $w / w)$ polymer was spread on the surface of the dispersion and mixed well to form the required 
gel. The strength of the prepared gel $(10 \mathrm{gm})$ is $0.2 \mathrm{~g}$ of propolis in each gram of the base. The summary of the newly prepared materials was presented in Table 1.

\subsection{Bioadhesive Study}

Bioadhesion studies were done using Chatillon apparatus for force measurement. [7] This method determines the maximum force and work needed to separate two surfaces in intimate contact [8]. The hydrogels $(0.1 \mathrm{~g})$ were homogeneously spread on a $1 \mathrm{~cm}^{2}$ glass disk and then the disks were fixed to the support of the tensile strength tester using double side adhesive. The gel was brought into contact with the contact with slice of dentin was established in order to imitate adhesion of the gel to the tooth structure, after a preset contact time (1 min) under contact strength $(0.5 \mathrm{~N})$ the 2 surfaces were separated at a constant rate of displacement $(1 \mathrm{~mm} / \mathrm{s})$. The strength was recorded as a function of the displacement, which allowed to determine the maximal detachment force, Fmax, and the work of adhesion, W, which was calculated from the area under the strength-displacement curve.

\subsection{Assessment of the Flavonoid Content in Propolis (Green Brazilian Propolis, Uruguayan Propolis and Australian Propols}

The Folin Ciocalteu assay determination was carried out by enzymatic method spectrophometrically, according to the procedures previously described and results are expressed as catechin equivalents (CE) [9].

\subsection{Determination of Gel pH}

One gram of the prepared gels was accurately weighed and dispersed in $10 \mathrm{ml}$ of purified water. The $\mathrm{pH}$ of the dispersions was measured using pH meter (HANNA instruments, HI8417, Portugal).

\subsection{Morphology of the Gels}

The samples were prepared by freezing in liquid nitrogen for $10 \mathrm{~min}$, and then were freezing-dried for $24 \mathrm{~h}$. The prepared samples were fractured in liquid nitrogen using a razor blade. The fractured samples were attached to metal stubs, and sputter coated with gold under vacuum for SEM. The interior and the surface morphology were observed in scanning electron microscope (SEM, Hitachi S4800, Japan).

\subsection{Gel Stability}

Stability of the gel formulations was also investigated. The organoleptic properties (color, odor), $\mathrm{pH}$, drug content, and release profiles of the gels stored at $20^{\circ} \mathrm{C}$ were examined on days $(0,15$, and 30$)$.

\subsection{Studies of Equilibrium Swelling in the Alternative Drug Delivery Systems}

The known weight nanodiamond-containing dry gels and naproxen-containing dry gels were immersed in $\mathrm{pH}$

Table 1. Gel formulation prepared in the study.

\begin{tabular}{|c|c|c|c|c|c|c|}
\hline Gel Formulation & & $\begin{array}{l}\text { Nanodiamond-Chitosan } \\
\text { (10\% ND:Ch) }\end{array}$ & $\begin{array}{l}\text { Propolis } \\
\text { Australian }\end{array}$ & $\begin{array}{l}\text { Propolis } \\
\text { Brazilian }\end{array}$ & $\begin{array}{l}\text { Propolis } \\
\text { Uruguyan }\end{array}$ & $\mathrm{pH}$ \\
\hline $\begin{array}{l}\text { Nanodiamond-Chitosan } \\
\text { (10\% ND:Ch) }\end{array}$ & Gel-1 & 5 & 0 & 0 & 0 & 6.14 \\
\hline $\begin{array}{l}\text { Nanodiamond-Chitosan } \\
\text { (10\% ND:Ch):Propolis (Australia) }\end{array}$ & Gel-2 & 5 & 1 & 0 & 0 & 6.35 \\
\hline $\begin{array}{l}\text { Nanodiamond-Chitosan } \\
\text { (10\% ND:Ch):Propolis (Brazil) }\end{array}$ & Gel-3 & 5 & 0 & 1 & 0 & 6.20 \\
\hline $\begin{array}{l}\text { Nanodiamond-Chitosan } \\
\text { (10\% ND:Ch):Propolis (Uruguya) }\end{array}$ & Gel-4 & 5 & 0 & 0 & 1 & 6.15 \\
\hline
\end{tabular}

Where: Hydrogels containing nanodiamond:chitosan (5\%) are synthesized and characterized. 
4.0, $\mathrm{pH} 9.0$ buffer solutions, respectively, and kept at $25^{\circ} \mathrm{C}$ for $48 \mathrm{~h}$ until equilibrium of swelling had been reached.

The swollen gels were taken out and immediately weighed with microbalance after the excess of water lying on the surfaces was absorbed with a filter paper. The equilibrium swelling ratio (SR) was calculated using the following equation:

$$
\mathrm{SR}=\left(\mathrm{W}_{\mathrm{s}}-\mathrm{W}_{\mathrm{d}}\right) / \mathrm{W}_{\mathrm{d}} \times 100 \%
$$

where $\mathrm{W}_{\mathrm{s}}$ and $\mathrm{W}_{\mathrm{d}}$ are the weights of the gels at the equilibrium swelling state and at the dry state, respectively. [9] Experiments were repeated 6 times for each gel specimen and mean value was obtained.

\subsection{Shear Bond Strength Tests for Dentin Bonding}

Extracted non-carious, intact, human molars stored in water containing a few crystals of thymol at $4^{\circ} \mathrm{C}$ were used within two months. Samples were checked before use for any damage caused by their removal. The roots of the teeth were removed with a separating disc and the occlusal enamel removed by grounding wet on 60-grit silicon carbide ( $\mathrm{SiC}$ ) paper. The teeth were embedded in PVC (Consjit Tubing, SA PVC, JHB, RSA) pipe containers with cold cure acrylic resin so that the grounded occlusal surfaces projected well above the resin. The 10 $\mathrm{mm}$ length pipes were put on a glass surface with one end blocked by the glass and the embedding done through the open end. Immediately after embedding the occlusal surfaces were ground wet with 180-grit followed by 600-grit $\mathrm{SiC}$ on a polishing machine to expose the superficial dentin. The samples were washed under a stream of tap water. A standardized zig (Ultradent ISO A2-70) with an internal diameter of $2.5 \mathrm{~mm}$ and height of $3 \mathrm{~mm}$ was used to shape the composite resin stud (SDR, Dentsply, CA, USA, Batch number 1105000609, Exp 2013-04). Two of these studs were then bonded to the polished dentin surface of each tooth via the bonding agent XP bond (Dentsply, New York, USA) using the following methodology: Groups C-E: Gels (1 - 4) (0.01 g, 10 seconds) + primer + Bonding immediately) or Groups K-N Gels (1 - 4) (0.01 g, 10 seconds) + Bonding immediately) The bonding agent contained: carboxylic acid modified dimethacrylate (TCB resin), phosphoric acid modified acrylate resin (PENTA), urethane dimetacrylate (UDMA), triethyleneglycol dimethacrylate (TEGDMA), 2-hydroxyethylmethacrylate (HEMA), butylated benzenediol (stabilizer), ethyl-4-dimethylamino-benzoate), camphorquinone, functionalized amorphous silica, t-butanol.

In this way were 80 teeth samples (each containing 2 studs) prepared and divided into 10 groups of 8 each, A-L (Table 2) and stored in a solution of artificial saliva. These groups were then treated as outlined in Table 2. After 24 hours one stud of each tooth was tested for shear bond strength and the other one after 3 months. An Instron Universal Testing Machine at a crosshead speed of $0.5 \mathrm{~mm} /$ minute was used to test the de-bonding strength. All data tests were analysed using the non-parametric ANOVA test.

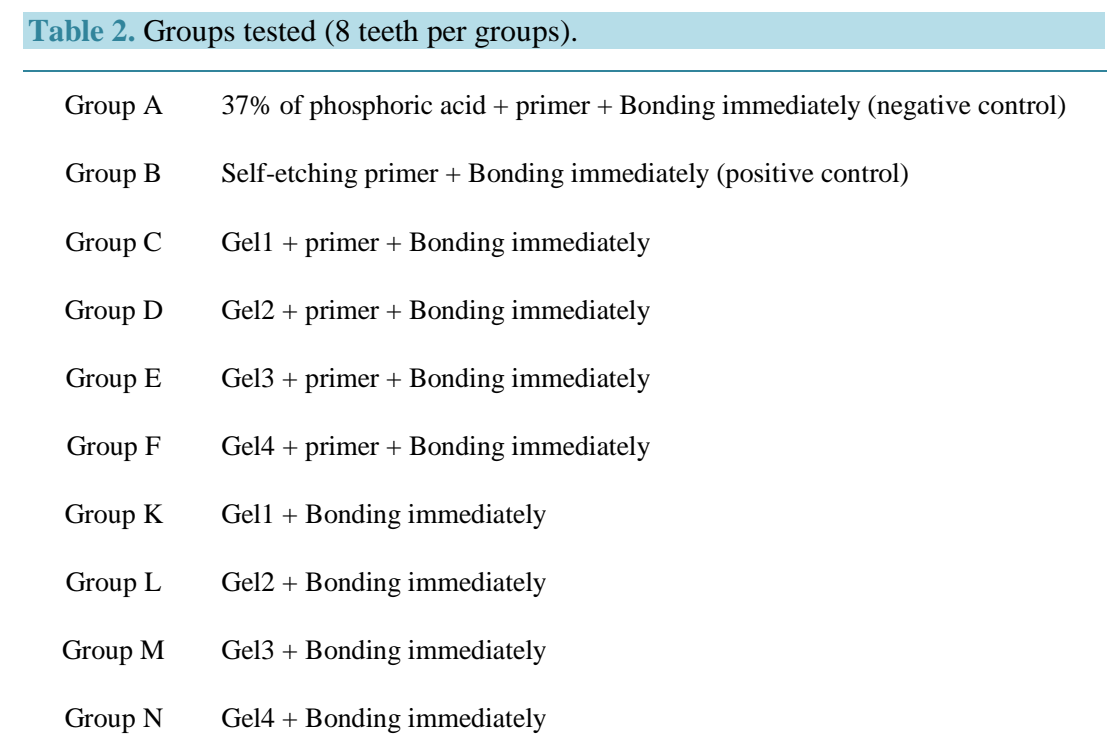




\section{Results}

The SEM images were obtained to characterize the microstructure of the freeze-dried propolis containing nanodiamond:chitosan gels and are presented in Figure 1. It could be seen that the gels displayed a homogeneously pore structure. It was thought that the micro-porous structure of the gels could lead to high internal surface areas with low diffusional resistance in the gels. The surfaces of the gels were also presented (Figure 1). The "skin" of the gels can be seen, and the collapse of the surface pores may be due to freeze-drying process.

The hydrogels remain in the cylindrical form after swelling. Compared with dry state hydrogels, the swollen state hydrogel volume display significant increases. Interestingly when comparison is made between the nanodiamond:chitosan hydrogel containing propolis of different origin versus the chitosan:propolis containing hydrogels the percentage of water uptake is not significanly different as summarized in Figure 2 suggesting that the materials are suitable for the proposed role as bioactive restorative materials.

Equilibrium swelling ratio (SR) of hydrogels exerts an influence on their release rates. The reduction in equilibrium swelling capacity is due to the formation of a tight network structure in high content. Environmental $\mathrm{pH}$ value has a large effect on the swelling behavior of these gels. From Figure 2 and our previously reported results [10] [11], it is clear that the SR value increases with the increase of $\mathrm{pH}$. Such $\mathrm{pH}$ dependent properties of the hydrogels come from the polyelectrolyte nature of chitosan segments in the hydrogel network. Namely, when the $\mathrm{pH}$ value of the buffer solution (pH 9.0) was far higher than the isoelectric point (PI) of GEL (PI 4.0 - 5.0),

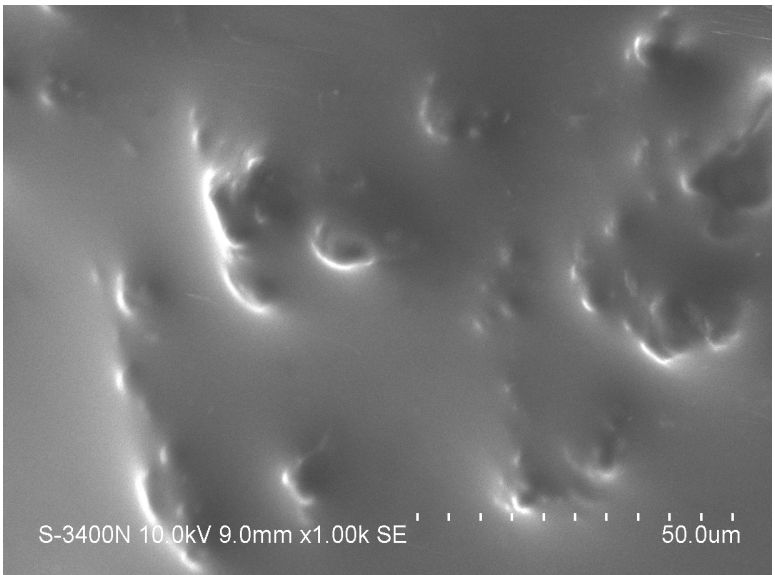

(a)

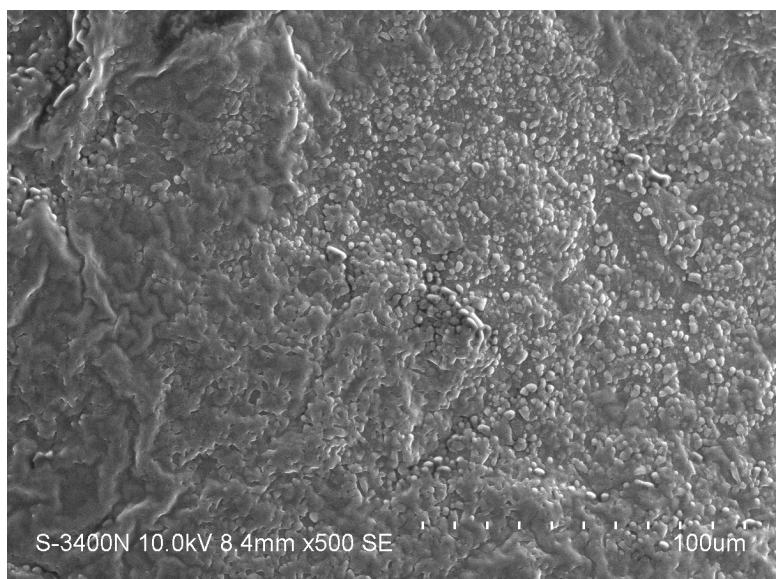

(c)

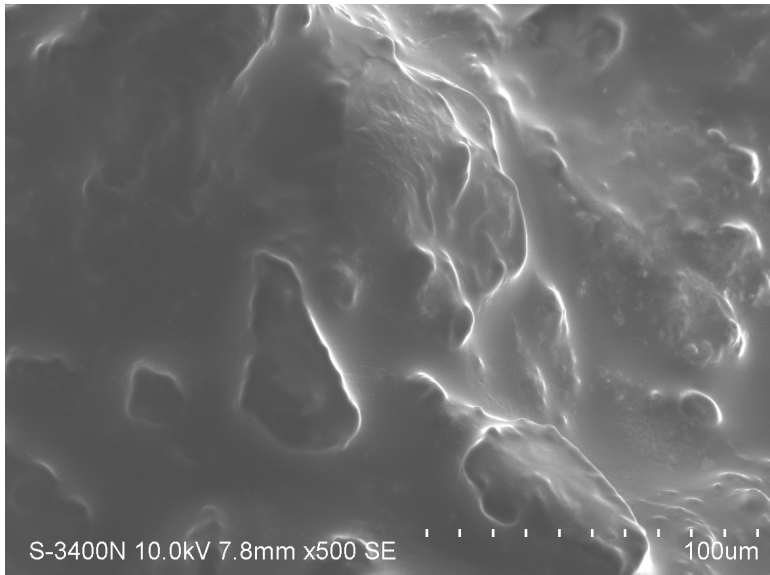

(b)

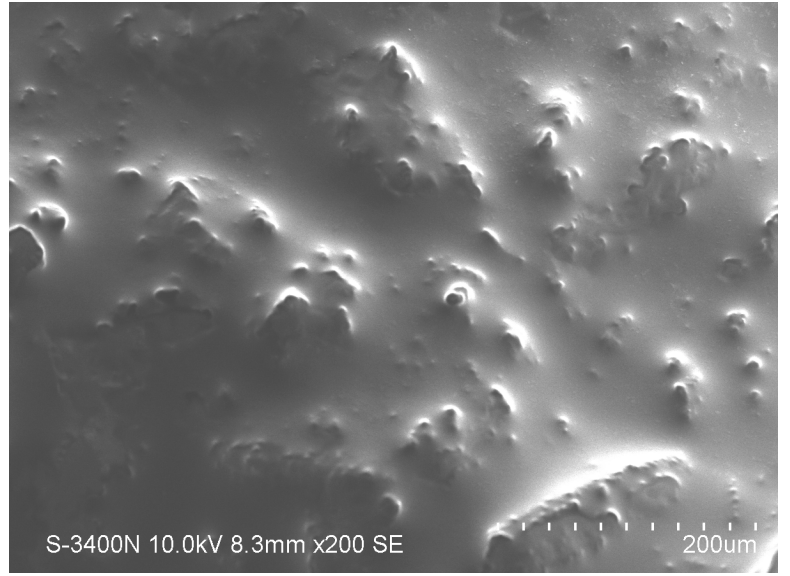

(d)

Figure 1. SEM photographs of interior morphology of the selected gels under investigation for (a) Chit/nanodiamond/ Propolis Uruguayan; (b) Chit/nanodiamond/Brazilian propolis; (c) Chit/nanodiamond/Propolis Australia; (d) Chit/nanodiamond. Studies of equilibrium swelling in various propolis containing nanodiamond: chitosan hydrogels (Gel $1-4)$ and comparison to propolis containing chitosan hydrogels. 


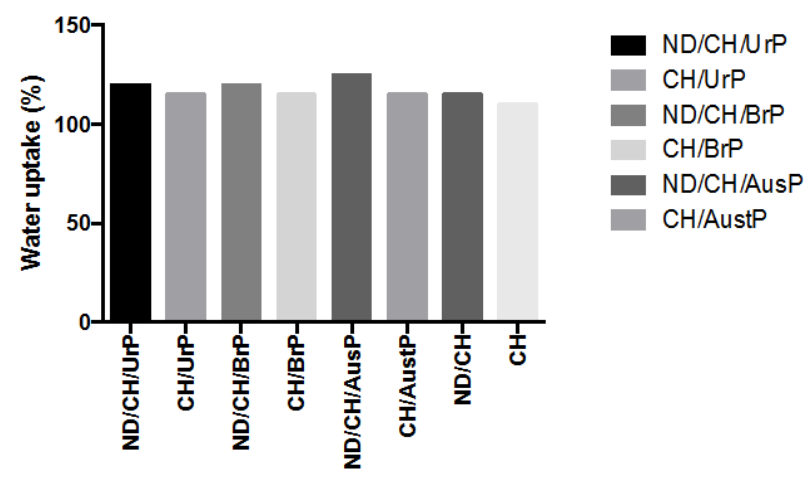

Figure 2. Comparison of water uptake percentage of the gels Gel-1 - Gel-4 ( $=6, \mathrm{p}<0.05)$ versus.

the carboxyl groups were de-protonized to carry negative charges, which made molecular chains repulsed to each other. The network became looser and it was easy for the water molecules to diffuse into the cross-linked network. According to above results, we believed that the propolis incorporation into the chitosan:nanodiamond containing hydrogel or into chitosan containing hydrogels influences the superposition of various effects, such as swelling property of hydrogels, the solubility of the drug and erosion property of matrix; it is not necessarily based on a single factor and therefore requires further investigation into the nature of the interaction of the bioactives in the propolis as well as nature of the interaction between nanodiamond and chitosan in the hydrogel.

\subsection{Shear Bond Strengths}

Figure 3 and Figure 4 gives the shear bond strength values (MPa) after 24 hours (Figure 3) and after 3 month of storage of samples in artificial saliva (Figure 4) using conventional dental adhesive systems such as 3 bottle etch/prime/bond system (standard 1) and 2 bottle self etch/prime and bond system (standard 2).

Mean shear bond strength values and difference between the groups are summarized in Figure 3 for bonding to dentin after 24 hours and in Figure 4 for bonding after 3 month. In general there was an increase in bond strength of the dentin treated with the propolis containing nanodiamond:chitosan hydrogels compared to the bond strength of the conventionally bonded teeth. An increase in the shear bond strength was also previously reported [11] for chitosan containing hydrogels. Interestingly the increase in bond strength was also observed in the groups of hydrogen peroxide exposed samples suggesting that there additional benefits associated with nanodiamond:chitosan:propolis system are in need of further investigations [12].

The results of this study suggests that the optimum results for the strengthening of dentin can be achieved throughout the immediate treatment with propolis:chitosan:nanodiamond with the increase of dentin bond strength. Also, impressively an almost immediately after the corresponding gel treatment and proceeding with bonding procedures is recommended with the significant increase in bond strength. The additional advantage of the system may suggest that, chitosan:propolis and nanodiamond interection with crystalline hydroxyapatite structure of the dentin layer increases the dentin bond strength observed especially in the case of the direct bonding between the hydrogel and the dentin interface. The increase of the bond strength is also attributed to the unique capacity of chitosan as well as biological properties of propolis to influence bioadhesion of the hydrogels and promote the formation of the reparative hydroxyapatite interface and therefore starting all important hydroxyapatite regeneration process making these type of designer bioactive hydrogels as important prototypes in development of bioactive restorative materials.

The additional benefit of using chitosan:antioxidant system as a bonding/pre-bonding to enamel and dentin system lies in its ability to show favorable immediate results in terms of bonding effectiveness as well as the durability of resin-dentin bonds for a prolonged time (up to 6 months) [13]. It is well documented that the hydrostatic pulpal pressure, the dentinal fluid flow and the increased dentinal wetness in vital dentin can affect the intimate interaction of certain enamel and dentin adhesives with dentinal tissue. There for the newly developed chitosan:antioxidant systems might at least be able to address the shortfalls in the current perspectives for improving bond durability through understanding factors affecting the long-term bonding performance of modern adhesives and addresses the current perspectives for improving bond durability. 


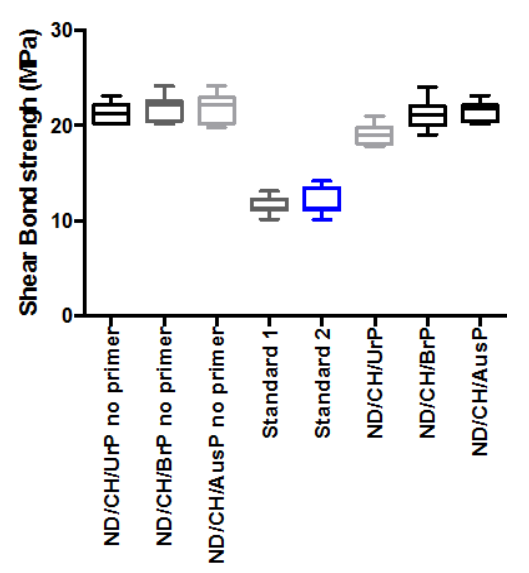

Figure 3. Shear bond strength of hydrogels after 24 hours of bonding to dentin.

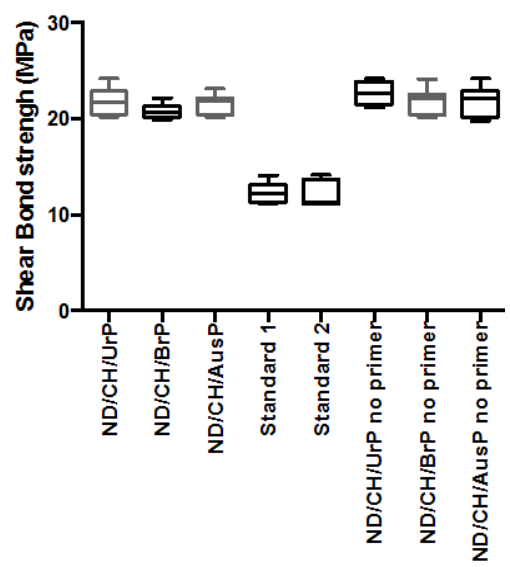

Figure 4. Shear bond strength of hydrogels after 3 month of bonding to dentin in artificial saliva.

\subsection{Propolis Extracts and Flavonoid Content of the Brazilian Green Propolis, Uruguyan Propolis and Australian Propolis Used in This Study}

On the basis of calibration curve for standard flavonoids, the concentration of these bioactive compounds in the hydroalcoholic extracts of the propolis sample used in the study was assessed. The content of flavonoids of the propolis was $1.73 \% \pm 0.35 \%$ (Green Brazilian Propolis), 1.65\% $\pm 0.25 \%$ (Uruguyan Propolis) and 1.28\% \pm $0.43 \%$ (Australian Propolis) which is consistent with the previously reported values for a hydroalcoholic extracts of the propolis in the literature [14]. The flavonoid content of the propolis extracts is the major contributing factor in biological activity of the propolis. The biological effects of flavonoids can be subdivided into 4 categories: 1) binding affinity to biological polymers, 2) binding of heavy metal ions, 3) catalyst of electron transport and 4) ability to scavenge free radicals [15]. The proposed mechanism of the propolis and its ability to mediate the inflammatory response in vivo have been previously explored in some details and suggested that the healing properties of propolis can be directly correlated with anti-oxidant potential as well as anti-inflammatory properties of flavonoids [16]. The work is currently on the way in our laboratories to evaluate the mechanism in vitro and in vivo in great details.

\subsection{Free Radical Defense Capability of the Prepared Nanodiamond:Chitosan Hydrogels}

The amount of uncontrolled ROS is the main cause of the inability of the healing process to continue, and therefore, it would be ideal to utilize the antioxidant capacity of the "designer hydrogels" to detect and be able to "fight the free radical excess". This has been assessed using a previously described model, where the HO radical 
can be generated from a reaction known as the biologic Fenton reaction, and this reaction requires the presence of $\mathrm{H}_{2} \mathrm{O}_{2}$ [17]. Bovine serum albumin (BSA), a completely water-soluble protein, was polymerized by hydroxyl radicals generated by the Fenton reaction system of $\mathrm{Fe}^{2+} /$ EDTA $/ \mathrm{H}_{2} \mathrm{O}_{2} /$ ascorbate [18]. As a result, the protein loses its water-solubility and the polymerized product precipitates. The decrease in the concentration of the water-soluble protein can easily be detected.

We considered it worthwhile to study the chitosan: as a "built-in defence mechanism" for the in vitro-generated free radical production and "site-specific" in vitro model counter reaction of the hydrogel. Therefore, we adopted a method for recording changes in the water solubility of the model protein, bovine serum albumin (BSA), exposed to free radicals generated by an inorganic chemical system. As clearly demonstrated by Figure 5 and Figure 6, upon exposure to standard $\mathrm{H}_{2} \mathrm{O}_{2}$ in the form of $\mathrm{Fe}^{2+} /$ EDTA/ $\mathrm{H}_{2} \mathrm{O}_{2} /$ ascorbate solution as a baseline determinate for free radical generation under "prototypical in vitro free radical damage", upon incorporation of the chitosan substituted hydrogels, the built-in antioxidant capacity and, therefore, free radical defence of the in vitro model have been activated and are of a significant value for which notice should be taken. This model represents the practical approach of in situ monitoring and testing of the amount of the free radical production and synergistic antioxidant defence of the system. Further investigations with fine-tuning of the system are currently on the way in our laboratory.

As clearly demonstrated by Figure 5 and Figure 6, upon exposure to standard $\mathrm{H}_{2} \mathrm{O}_{2}$ in the form of $\mathrm{Fe}^{2+}$ / EDTA/ $\mathrm{H}_{2} \mathrm{O}_{2}$ /ascorbate solution as a baseline determinate for free radical generation under "prototypical in vitro free radical damage", upon incorporation of the chitosan substituted hydrogels, the built-in antioxidant capacity and, therefore, free radical defence of the in vitro model have been activated and are of a significant value for which notice should be taken. This model represents the practical approach of in situ monitoring and testing of the amount of the free radical production and synergistic antioxidant defence of the system. Further investigations with fine-tuning of the system are currently on the way in our laboratory.

\subsection{Bio-Adhesion in Vitro Model}

The higher adhesiveness of the gels is desired to maintain intimate contact with the tooth structure, and the results are summarized in Table 3. Nanodiamond:chitosan hydrogels showed the highest adhesive force and work of adhesion, which can be expected, because of the well-known intrinsic bioadhesive properties of chitosan

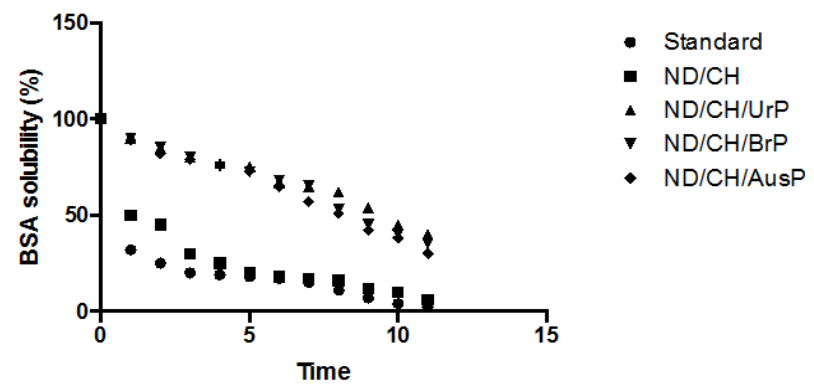

Figure 5. Free radical defence after 24 hours of storage of the materials.

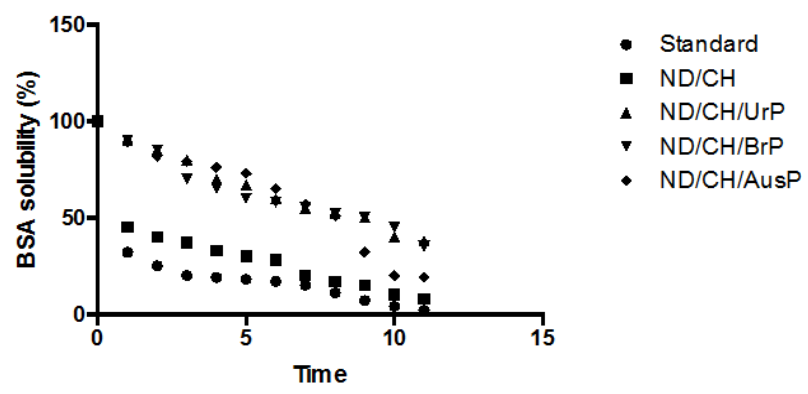

Figure 6. Free radical defence after 24 hours of storage of the materials. 
Table 3. In vitro bio-adhesion test.

\begin{tabular}{ccc}
\hline Hydrogel & $\begin{array}{c}\text { Adhesive Force }(\mathrm{N}) \\
\pm \text { SD (Dentin) }\end{array}$ & $\begin{array}{c}\text { Work of Adhesion }(\mathrm{N} \cdot \mathrm{cm}) \\
\pm \mathrm{SD} \text { (Dentin) }\end{array}$ \\
\hline Gel 1 & $1.18 \pm 0.35$ & $2.51 \pm 0.42$ \\
Gel 2 & $1.78 \pm 0.41$ & $6.01 \pm 0.25$ \\
Gel 3 & $1.87 \pm 0.38$ & $6.34 \pm 0.24$ \\
Gel 4 & $1.76 \pm 0.32$ & $5.98 \pm 0.28$ \\
\hline
\end{tabular}

[19]-[22]. The adequate water absorption capacity together with the cationic nature, which promotes binding to the negative surface of dentin structure, can also interpret these results.

Propolis containing hydrogels have shown the highest adhesive force and work of adhesion in comparison the nanodiamond:chitosan hydrogels which are not containing the propolis aditives. This can be expected, because of the well-known intrinsic bio-adhesive properties of chitosan and propolis. The adequate water absorption capacity together with the cationic nature, which promotes binding to the negative surface of skin and the dentin structure, can also be used to interpret these results. It is well documented that hydration of the polymer causes mobilization of the polymer chains and, hence, influences polymeric adhesion [23]. Appropriate swelling is important to guarantee adhesivity; however, over-hydration can form slippery non-adhesive hydrogels [23]. In addition, for the molecular arrangement of the polymeric chains, which are present in the new hydrogels, such as with propolis, the correlation between the force and work of adhesion is noticeable for all. Further experiments are to be conducted on skin samples to further evaluate the bio-adhesive capacity of the designer hydrogels.

\section{Conclusion}

The materials were prepared and tested in order to quantify the effects of functional designer biomaterials on the dentin bond strength of a composite, capacity to act in the protective capacity against free radical damage and evaluated the bio-adhesive capacity of the materials in the two separate "in vitro" systems. Within the limitations of the study design, such as reliable in vitro model system, capable of providing insight into the molecular origin of the hydrogel performance and interactions, chitosan-based hydrogels are suitable materials for functional restorative in vitro. Cytotoxicity data are currently being evaluated in our laboratory.

\section{Acknowledgements}

VTP would like to acknowledge the support of VTPCHEM PTY LTD.

\section{References}

[1] Schrand, A., Hens, S.A.C. and Shenderova, O. (2009) Nanodiamond Particles: Properties and Perspectives for Bioapplications. Critical Reviews in Solid State and Materials Sciences, 34, 18-74. http://dx.doi.org/10.1080/10408430902831987

[2] Danilenko, V.V. (2004) On the History of the Discovery of Nanodiamond Synthesis. Physics of the Solid State, 46, 595-599. http://dx.doi.org/10.1134/1.1711431

[3] Shenderova, O.A. and Hens, S.A.C. (2010) Detonation Nanodiamond Particles Processing, Modification and Bioapplications. In: Ho, D., Ed., Nanodiamonds: Applications in Biology and Nanoscale Medicine, Springer, New York, 79-116. http://dx.doi.org/10.1007/978-1-4419-0531-4_4

[4] Ahn, M.R., Kunimasa, K., Ohta, T., Kumazawa, S., Kamihira, M., Kaji, K., Uto, Y., Hori, H., Nagasawa, H. and Nakayama, T. (2007) Suppression of Tumor-Induced Angiogenesis by Brazilian Propolis: Major Component Artepillin C Inhibits in Vitro Tube Formation and Endothelial Cell Proliferation. Cancer Letters, 252, 235-243. http://dx.doi.org/10.1016/j.canlet.2006.12.039

[5] Amaral, R.C., Gomes, R.C., Rocha Dos Santos, W.M., Abreu, S.L.R. and Santos, V.R. (2006) Periodontitis Treatment with Brasilian Green Propolis Gel. Pharmacologyonline, 3, 336-341.

[6] Bankova, V., Christov, R., Kujumgiev, A., Marcucci, M.C. and Popov, S. (1995) Chemical Composition and Antibacterial Activity of Brazilian Propolis 857. Zeitschrift fur Naturforschung, Section C, Biosciences, 50, 167-172.

[7] Bankova, V.S., Christov, R., Popov, S., Marcucci, M.C., Tsvetkova, I. and Kujumgiev, A. (1999) Antibacterial Activi- 
ty of Essential Oils from Brazilian Propolis. Fitoterapia, 70, 190-193. http://dx.doi.org/10.1016/S0367-326X(98)00045-8

[8] Bankova, V., Popova, M. and Trusheva, B. (2007) Plant Origin of Propolis: Latest Developments and Importance for Research and Medicinal Use, In: Marghitas, L.A. and Dezmirean, D., Eds., Apicultura-De la stiinta la agribusiness si apiterapie, Editura Academic Pres, Cluj Napoca, 40-46.

[9] Aoi, W., Hosogi, S., Niisato, N., Yokoyama, N., Hayata, H., Miyazaki, H., Kusuzaki, K., Fukuda, T., Fukui, M., Nakamura, N. and Marunaka, Y. (2013) Improvement of Insulin Resistance, Blood Pressure and Interstitial pH in Early Developmental Stage of Insulin Resistance in OLETF Rats by Intake of Propolis Extracts. Biochemical and Biophysical Research Communications, 432, 650-653. http://dx.doi.org/10.1016/j.bbrc.2013.02.029

[10] Perchyonok, V.T., Reher, V., Zhang, S.M., Basson, N.J. and Grobler, S.R. (2015) Bioinspired-Interpenetrating Network (IPNs) Hydrogel (BIOF-INPs) and TMD in Vitro: Bioadhesion, Drug Release and Build in Free Radical Detection and Defense. Open Journal of Stomatology, 5, 53-61. http://dx.doi.org/10.4236/ojst.2015.53008

[11] Perchyonok, V.T., Reher, V., Zhang, S.M., Basson, N.J. and Grobler, S.R. (2015) Bioactive-Functionalized Interpenetrating Network Hydrogel (BIOF-IPN): A Novel Biomaterial Transforming the Mechanism of Bio-Repair, Bio-Adhesion and Therapeutic Capability_An in Vitro Study. Journal of Interdisciplinary Medicine and Dental Science, 3, 1.

[12] Perchyonok, V.T., Zhang, S.M., Basson, N.J. and Grobler, S.R. (2014) Evaluation of Tetracycline Containing Chitosan Hydrogels as Potential Dual Action Bio-Active Restorative Materials Capable of Wound Healing: In Vitro Approach. Biointerface Research in Applied Chemistry, 4, 843-849.

[13] Perchyonok, V.T., Reher, V., Zhang, S.M., Ward, M. and Grobler, S.R. (2014) Microwave Assisted Prepared Interpenetrating Hydrogels from Guar-Gum: Chitosan IPN and Guar Gum Hydrogels as Novel Functional Materials: Bonding, Antioxidant and Bioactivity. Biointerface Research in Applied Chemistry, 4, 850-856.

[14] Perchyonok, V.T., Reher, V., Zhang, S.M., Grobler, S.R. and Basson, N.J. (2014) Evaluation of Nystatin Containing Chitosan Hydrogels as Potential Dual Action Bio-Active Restorative Materials: In Vitro Approach. Journal of Functional Materials, 5, 259-272. http://dx.doi.org/10.3390/jfb5040259

[15] Perchyonok, V.T., Zhang, S.M. and Grobler, S.R. (2014) IPNs from Cyclodextrin: Chitosan Antioxidants: Bonding, Bioadhesion, Antioxidant Capacity and Drug Release. Journal of Functional Materials, 5, 183-196.

[16] Hu, Q.L., Li, B.Q., Wang, M. and Shen, J.C. (2004) Preparation and Characterization of Biodegradable Chitosan/Hydroxyapatite Nanocomposite Rods via in Situ Hybridization: A Potential Material as Internal Fixation of Bone Fracture. Biomaterials, 25, 779-785. http://dx.doi.org/10.1016/S0142-9612(03)00582-9

[17] Perchyonok, V.T., Reher, V., Zhang, S.M., Grobler, S.R., Oberholzer, T.G. and Ward, M. (2014) Insights and Relative Effect of Aspirin, Naproxen and Ibuprofen Containing Hydrogels: From Design to Performance as a Functional Dual Capacity Restorative Material and Build in Free Radical Defence: In-Vitro Studies. Open Journal of Stomatology, 4, 73-83. http://dx.doi.org/10.4236/ojst.2014.42013

[18] Perchyonok, V.T., Zhang, S.M., Basson, N.J., Grobler, S.R., Oberholzer, T.G. and Ward, M. (2014) Insights into Functional Erythromycin/Antioxidant Containing Chitosan Hydrogels as Potential Bio-Active Restorative Materials: Structure, Function and Antimicrobial Activity. Advanced Techniques in Biology and Medicine, 2, 1.

[19] Perchyonok, V.T., Zhang, S.M., Grobler, S.R., Oberholzer, T.G. and Ward, M. (2014) Insights into and Relative Effect of Chitosan-Krill Oil, Chitosan-H-Aspirin, Chitosan-H-Krill Oil-Nystatin and Chitosan-H-Krill Oil-Aspirin-Nystatin on Dentin Bond Strength and Functional Drug Delivery Capacity: In-Vitro Studies. European Journal of General Dentistry, 3, 57-65. http://dx.doi.org/10.4103/2278-9626.126214

[20] Perchyonok, V.T., Reher, V., Zhang, S.M., Grobler, S.R., Oberholzer, T.G. and Ward, M. (2014) Insights into Functional Tea Infused-Chitosan Hydrogels as Potential Bio-Active Restorative Materials. European Journal of General Dentistry, 3, 22-28. http://dx.doi.org/10.4103/2278-9626.126205

[21] Perchyonok, V.T., Reher, V., Zhang, S.M., Oberholzer, T.G., Ward, M. and Grobler, S.R. (2014) Chitosan: Vitamin C Containing Hydrogels as a Prototype Functional Prolonged Pain Management Restorative Material In-Vitro Studies. Open Jounal of Stomatology, 4, 389-401. http://dx.doi.org/10.4236/ojst.2014.48053

[22] Perchyonok, V.T., Zhang, S.M., Basson, N.J., Grobler, S., Oberholzer, T.G. and Ward, M. (2013) Insights into Functional Tetracycline/Antioxidant Containing Chitosan Hydrogels as Potential Bio-Active Restorative Materials: Structure, Function and Antimicrobial Activity. Open Journal of Stomatology, 3, 75-82. http://dx.doi.org/10.4236/ojst.2013.31014

[23] Perchyonok, V.T., Zhang, S.M. and Oberholzer, T.G. (2013) Protective Effect of Conventional Antioxidant ( $\beta$-Carotene, Resveratrol and Vitamin E) in Chitosan-Containing Hydrogels against Oxidative Stress and Reversal of DNA Double Stranded Breaks Induced by Common Dental Composites: In-Vitro Model. The Open Nanoscience Journal, 7, 1-7. http://dx.doi.org/10.2174/1874140101307010001 